\title{
Maximum-likelihood implementation of the tip-of-the-red-giant-branch method
}

\author{
Dmitry Makarov \\ Special Astrophysical Observatory, Russian Academy of Sciences, N. Arkhyz, KChR, 369167, \\ Russia \\ email: dim@sao.ru
}

\begin{abstract}
The luminosity of the tip of the red giant branch (TRGB) provides an excellent measure of galaxy distances and is easily determined in the resolved images of nearby galaxies observed with the Hubble Space Telescope (HST). We use a maximum-likelihood algorithm to locate the TRGB in galaxy colour-magnitude diagrams. The algorithm is optimized by introducing reliable photometric errors and a completeness characterization determined based on artificial-star experiments. The program has been tested extensively using Monte Carlo simulations, artificial galaxies, and a sample of nearby dwarf galaxies observed with the HST's WFPC2 and ACS cameras. Our procedure is shown to be reliable, yield good accuracy, and does not introduce any systematic errors. The methodology is especially useful in cases in which the TRGB approaches the photometric limit and/or the RGB is poorly populated.
\end{abstract}

Keywords. methods: data analysis, galaxies: distances and redshifts

\section{Introduction}

The tip of the red giant branch (TRGB) is the most effective distance indicator to study the distribution of nearby galaxies. The sharp cut-off in the luminosity function of the red-giant stars is determined by a violent flash of the helium core in low-mass stars. The break is comparably bright, $M_{I} \sim-4$ mag. It is characterized by a weak age-metallicity dependence in the $I$ band. The TRGB method is comparable in accuracy to the Cepheid period-luminosity distance indicator (Sakai et al. 1993; Bellazzini et al. 2001; Rizzi et al. 2007). In addition, old stellar populations enable application of the TRGB method to galaxies of any morphological type. Because old stars are wide-spread across galaxies, we can exclude star-forming regions from the analysis to avoid problems with extinction in the host galaxy. Application of the TRGB method does not require long or complicated observations. All these conditions make it possible to estimate distances to a large set of galaxies using the TRGB method.

\section{The TRGB method}

Lee et al. (1993) developed the first quantitative method for detection of the jump in the luminosity function of red giants. They used a standard edge-detection algorithm with the zero-sum Sobel kernel $[-2,0,+2]$, which allows one to find the position of the greatest changes in a stellar distribution. They estimated the typical error at $0.1-0.2$ mag. Sakai et al. (1993) improved the quantitative approach to estimating the position of the TRGB using an adaptive edge-detection filter, which takes into account errors in the stellar photometry in a galaxy. Alternatively, Méndez et al. (2002) used a maximumlikelihood analysis for TRGB detection. They used a simple, broken-power-law model to describe the luminosity function. 

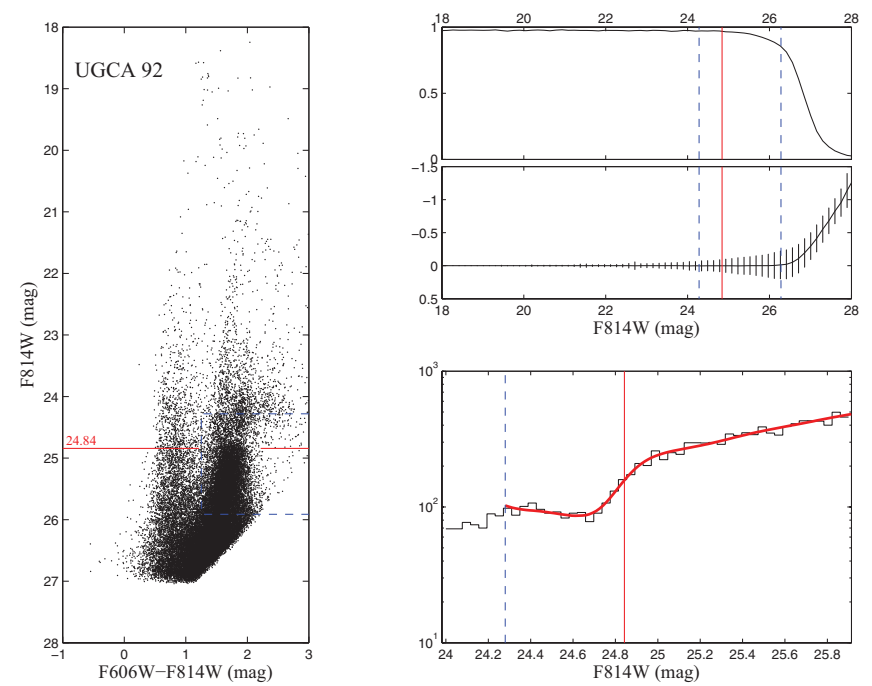

Figure 1. Illustration of the maximum-likelihood implementation of the TRGB method using the example of UGCA 92 (Makarova et al. 2012). The colour-magnitude diagram is shown in the left panel. The top right-hand panel shows the completeness, photometric errors and the dispersion in the errors (vertical bars) versus HST/ACS F814W magnitude. The bottom right-hand panel shows a histogram of the F814W luminosity function. The resulting model luminosity function convolved with photometric errors and incompleteness is displayed as a thick solid line with a jump at the position of the TRGB.

Both approaches have their strengths and weaknesses. The edge-detection algorithm is model-independent, while the quality of the maximum-likelihood approach depends on the reliability of the model luminosity function. On the other hand, edge detection tries to differentiate a discrete set of points that needs to be smoothed, which leads to specific noise and the result depends on the smoothing properties. The maximum likelihood uses all detected stars for analysis and its behaviour is regular.

Makarov et al. (2006) improved the maximum-likelihood method of Méndez et al. (2002) by implementing the use of reliable photometric errors and a completeness function based on artificial-star experiments. Artificial-star tests are the only accurate way to estimate photometric errors, the effects of blending and the photometric limit (Dolphin 2002). This approach allows us to take into account systematic errors introduced by crowding effects, Malmquist bias and incompleteness. The program was extensively tested using Monte Carlo simulations, artificial galaxies, and a sample of nearby dwarf galaxies observed with the Hubble Space Telescope (HST)'s WFPC2 and ACS cameras. The procedure was shown to be reliable, yield good accuracy, and does not introduce any systematic errors. This technique is especially useful in cases where the TRGB approaches the photometric limit and/or the RGB is poorly populated in the colour-magnitude diagram. Fig. 1 illustrates the distance determination using the method of Makarov et al. (2006). The zero point of the TRGB was calibrated by Rizzi et al. (2007) using the horizontal branch, yielding a statistical accuracy of $1 \%$. It was found that the TRGB zero point is in good agreement with the Cepheid distance scale.

Over the past decade, we have gained significant experience in distance determination to nearby galaxies with the HST. One of the most important tasks involves cleaning the RGB from the influence of young stellar populations, because the latter can significantly affect the luminosity function. Often, the luminosity distribution of the asymptotic giant branch cannot be represented by simple power law. In that case, it is necessary to either 


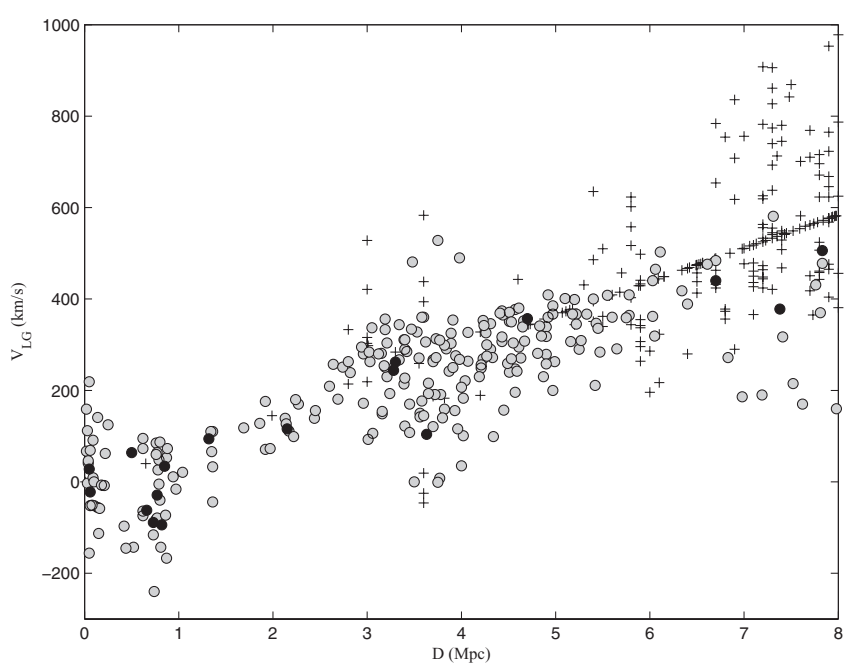

Figure 2. Hubble diagram for galaxies within $8 \mathrm{Mpc}$. Galaxies with TRGB distance determinations are shown as light grey circles. Black dots correspond to galaxies with Cepheid distances and crosses represent objects with distance estimates based on other methods.

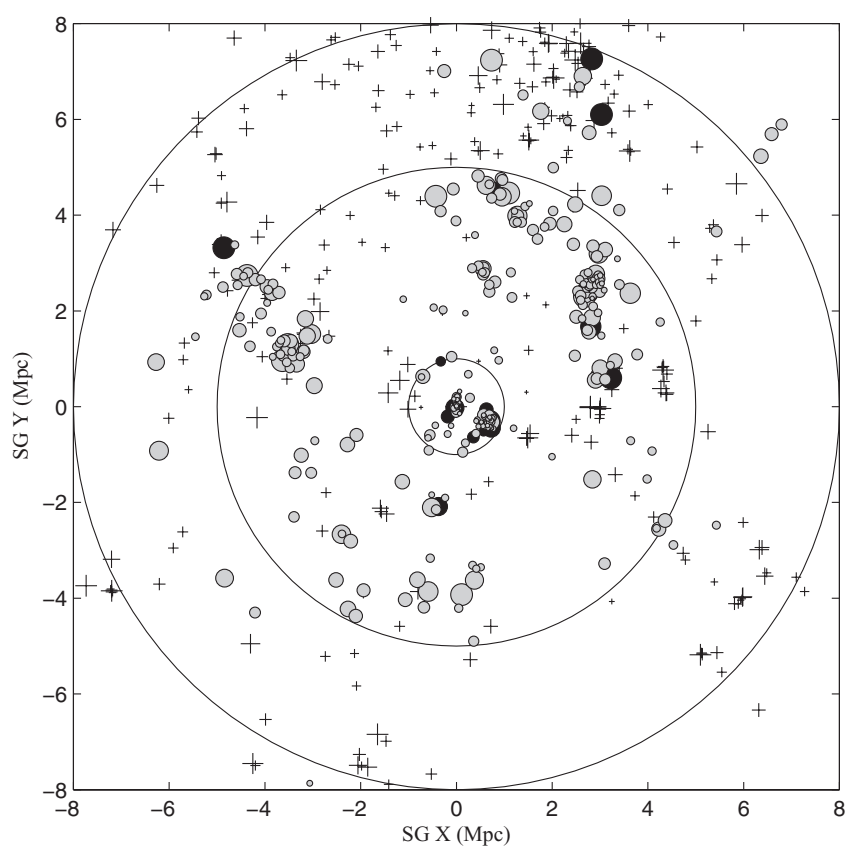

Figure 3. Map of the nearby galaxy distribution $(D<8 \mathrm{Mpc})$ projected onto the Supergalactic plane. Light grey circles correspond to galaxies with TRGB distances, black dots show objects with Cepheids distances, and crosses represent other methods of distance determination. The size of the symbols corresponds to the luminosity of the respective galaxy.

limit the domain or use more complicated functions. These effects should be taken into account to avoid systematic biases in distance determination.

The precision of the TRGB method is comparable with that achieved using Cepheids. It is extremely effective on scales of up to 5-10 Mpc. The overwhelming majority of precise distances below $6 \mathrm{Mpc}$ are based on TRGB distance estimates. This is illustrated 
in the distance-velocity diagram in Fig. 2. Only $6 \%$ of distances within $6 \mathrm{Mpc}$ from our Galaxy were measured using the Cepheid period-luminosity relation. Currently, TRGB distances are known for some 270 galaxies. The 3D map of luminous matter in the Local Volume (see Fig. 3) is based on homogeneous data obtained mostly based on the TRGB method.

\section{Conclusion}

Our experience with TRGB distance determination allows us to conclude that this method is very effective to study the distribution of matter out to $5-10 \mathrm{Mpc}$. The maximum-likelihood method allows us to estimate distances even near the photometric limit and for poorly populated RGBs. Because the TRGB method is based on tracers from the old stellar population, it can be applied to galaxies of any type. In case of a 'polluted' RGB, one must be very careful in one's selection of stars and avoid the central parts of a galaxy and regions of active star formation. Often, the luminosity function of the asymptotic giant branch is more complicated than a single power law. These effects should be taken into account to avoid systematic biases in distance determinations.

\section{Acknowledgements}

This work was supported by the Russian Foundation for Basic Research (RFBR), grant 11-02-00639, and Russian-Ukrainian RFBR grant 11-02-90449. We acknowledge support from the Ministry of Education and Science of the Russian Federation under contract 14.740.11.0901 and proposal 2012-1.5-12-000-1011-004.

\section{References}

Bellazzini, M., Ferraro, F., \& Pancino, E. 2001, ApJ, 556, 635

Dolphin, A. E. 2002, MNRAS, 332, 91

Lee, M. G., Freedman, W. L., \& Madore, B. F. 1993, ApJ, 417, 553

Makarov, D., Makarova, L., Rizzi, L., Tully, R. B., Dolphin, A. E., Sakai, S., \& Shaya, E. J. 2006, AJ, 132, 2729

Makarova, L., Makarov, D., \& Savchenko, S. 2012, MNRAS, 423, 294

Méndez, B., Davis, M., Moustakas, J., Newman, J., Madore, B. F., \& Freedman, W. L. 2002, $A J, 124,213$

Rizzi, L., Tully, R. B., Makarov, D., Makarova, L., Dolphin, A. E., Sakai, S., \& Shaya, E. J. 2007, ApJ, 661, 815

Sakai, S., Madore, B. F., \& Freedman, W. L. 1996, ApJ, 461, 713 